\title{
Ischemic Stroke in a Patient With Polyneuropathy, Organomegaly, Endocrinopathy, Monoclonal Protein, and Skin Changes Syndrome Treated With Lenalidomide
}

\author{
Soujanya Sodavarapu ${ }^{1}$, Arshian Mahajan ${ }^{2}$ \\ 1. Internal Medicine, San Joaquin General Hospital, French Camp, USA 2. Family Medicine, San Joaquin General \\ Hsopital, French Camp, USA
}

Corresponding author: Soujanya Sodavarapu, ssodavar@sigh.org

\begin{abstract}
Polyneuropathy, organomegaly, endocrinopathy, monoclonal protein, and skin changes (POEMS) syndrome is an uncommon multisystemic disease associated with plasma cell dyscrasia. Due to the disease's rarity and an even rarer presentation of stroke in afflicted patients, a direct association between POEMS syndrome and stroke remains ambiguous. Thrombocytosis, hyperfibrinogenemia, and increased levels of inflammatory cytokines occur in this disease, which can predispose patients to thromboembolic events. Immunomodulators can also enhance thrombosis, the chances of which increase when they are combined with dexamethasone. We present a case of a 28 -year-old patient with an ischemic stroke, which may have been triggered by the combination of POEMS syndrome-associated vasculitis and the thrombogenic nature of lenalidomide-dexamethasone therapy.
\end{abstract}

Categories: Neurology, Oncology

Keywords: poems syndrome, lenalidomide

\section{Introduction}

Polyneuropathy, organomegaly, endocrinopathy, monoclonal protein, and skin changes (POEMS) syndrome is an uncommon multisystemic disease associated with plasma cell dyscrasia [1]. Thrombocytosis, hyperfibrinogenemia, and increased levels of inflammatory cytokines occur in this disease, and hence the patient may be susceptible to thromboembolic events. However, its association with cerebrovascular events is still being evaluated. Lenalidomide, in combination with dexamethasone, is an effective treatment for POEMS syndrome but poses a high risk of venous thromboembolism [2]. We present a case of a 28-year-old male with ischemic stroke who was diagnosed with POEMS syndrome and was treated with lenalidomidedexamethasone combination therapy.

Received 07/01/2020 Review began $07 / 03 / 2020$ Review ended 07/10/2020 Published 07/22/2020

\section{() Copyright 2020}

Sodavarapu et al. This is an open access article distributed under the terms of the Creative Commons Attribution License CC-BY 4.0., which permits unrestricted use, distribution, and reproduction in any medium, provided the original author and source are credited.

\section{Case Presentation}

A 28-year-old male with a history of hypertension and aortic stenosis presented with paresthesia of the left foot, stiffness in the lower back and neck, and weight loss of 15 pounds for the past few months. His vitals were stable at presentation. On physical examination, the cranial nerves were intact, while strength and sensation in extremities were normal, except for bilateral foot drop and decreased lower extremity tone. Xrays of the lumbar spine showed sclerosis of the L4-L5 vertebral bodies and small sclerotic densities in the left iliac bone. An MRI showed a large sclerotic lesion at L5 and small sclerotic lesions in the bilateral iliac. A CT scan of the torso showed prominent bilateral axillary and mediastinal lymph nodes, hepatomegaly, splenomegaly, retroperitoneal and iliac lymph node enlargement, and widespread sclerotic foci in the bones. A subsequent ultrasound-guided left inguinal biopsy showed atypical lymphoid proliferation. Moreover, a CT-guided retroperitoneal lymph node biopsy showed mild kappa-predominant plasmacytosis, with faint monoclonal lambda chains on protein electrophoresis. A lymph node biopsy showed an abundance of plasma cells that were polytypic for kappa and lambda. This was in line with high kappa (56.3 mg/L) and lambda $(31.5 \mathrm{mg} / \mathrm{L})$ hematological test values, with a ratio of $1: 79$. Vascular endothelial growth factor (VEGF) levels were elevated at $1,156 \mathrm{pg} / \mathrm{mL}$, while electromyography studies were consistent with severe axonal loss and demyelination. The patient was subsequently diagnosed with POEMS syndrome.

The patient was treated with radiotherapy and lenalidomide with dexamethasone. VEGF levels improved to normal $(231 \mathrm{pg} / \mathrm{mL})$. However, he developed a headache and expressive aphasia after three months on lenalidomide. A CT brain revealed an acute non-hemorrhagic infarct in the left temporal cortex (Figure 1), while magnetic resonance angiography showed no signs of atherosclerosis in the cranial vessels. His stroke was presumed to be due to the thrombogenic effect of POEMS syndrome with superimposed thrombogenesis from lenalidomide, which was subsequently stopped while he received aspirin $81 \mathrm{mg}$ and atorvastatin $40 \mathrm{mg}$ daily. He was started on carfilzomib and dexamethasone but did not experience clinical improvement. He is being considered for melphalan and autologous stem cell transplantation. 


\section{Cureus}

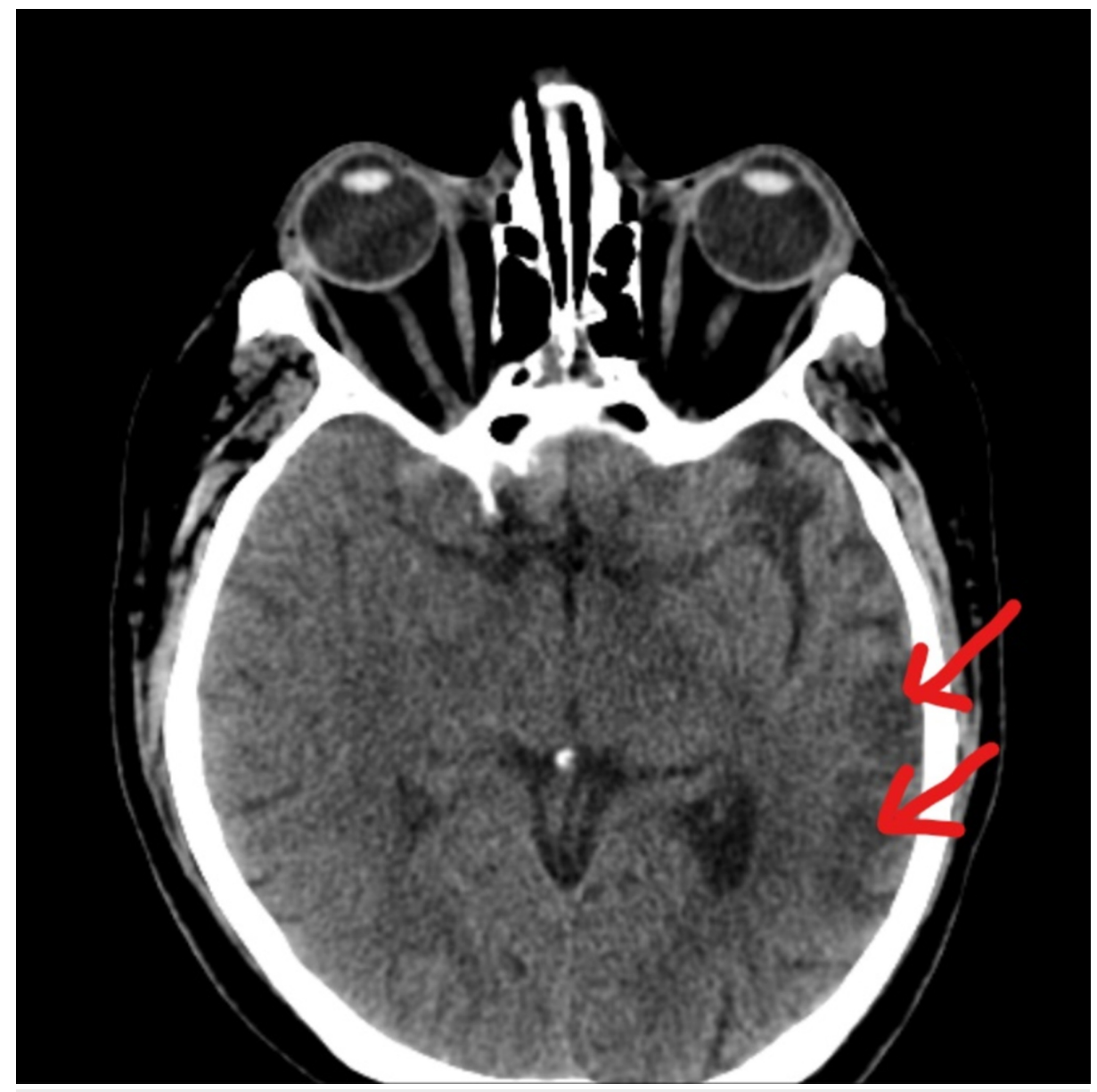

FIGURE 1: CT of the brain

The image shows an acute non-hemorrhagic infarct in the left temporal cortex (arrows)

CT: computed tomography

\section{Discussion}

POEMS syndrome is a rare, life-threatening, and disabling plasma cell disorder where the acronym stands for polyneuropathy, organomegaly, endocrinopathy, monoclonal protein, and skin changes [1]. Though the pathophysiology underlying the disorder remains unknown, it is postulated to be due to various proinflammatory growth factors and cytokines that are thought to stimulate VEGF expression, which likely plays a vital role in the pathogenesis of the disease [2]. Its presentation varies from person to person and, due to its diverse clinical manifestations, especially when presented to a primary care provider, can have a longer lead time for diagnosis ranging from 13-18 months, and could be even misdiagnosed as other disorders such as chronic inflammatory demyelinating polyneuropathy, amyloidosis, and multiple myeloma [2]. Diagnosis of POEMS syndrome requires the fulfillment of two mandatory criteria, one or more major criteria, and one or more minor criteria (Table 1) [3]. 


\section{Cureus}

\section{Criteria,}

symptoms,

and

Description

diagnosis

Mandatory criteria

1. Polyneuropathy (typically demyelinating). 2. Monoclonal plasma cell-proliferation disorder (almost always $\lambda$ )

Major

criteria

1. Sclerotic bone lesions. 2. Castleman disease. 3. Vascular endothelial growth factor elevation

Minor

criteria

1. Organomegaly (splenomegaly, hepatomegaly, or lymphadenopathy). 2. Extravascular volume overload (edema, pleural effusion, or ascites). 3. Endocrinopathy (adrenal, thyroid, pituitary, gonadal, parathyroid, pancreatic). 4. Skin changes (hyperpigmentation, hypertrichosis, glomeruloid hemangiomata, plethora, acrocyanosis, flushing, and white nails). 5. Papilledema. 6. Thrombocytosis/polycythemia

Other

symptoms

and signs

Clubbing, weight loss, hyperhidrosis, pulmonary hypertension/restrictive lung disease, thrombotic diatheses, diarrhea, low vitamin B12 values

Diagnosis

of POEMS

syndrome

Requires the fulfillment of two mandatory criteria $+\geq 1$ major $+\geq 1$ minor criteria

TABLE 1: Diagnostic criteria for POEMS syndrome

POEMS syndrome: polyneuropathy, organomegaly, endocrinopathy, monoclonal protein, and skin changes syndrome

Due to the disease's rarity and an even rarer presentation of stroke in afflicted patients, a direct association between POEMS syndrome and stroke remains ambiguous. Dupont et al. have described a case series of 208 patients, of which 19 patients (9.2\%) developed cerebral infarction with a median age of 53 years (range: 3677 years) [4]. Acute vascular angiopathy and macroangiopathy from the increased inflammatory growth factors and VEGF are assumed to cause vasculitis and vascular anomaly in these patients $[5,6]$. High levels of proinflammatory cytokines with hyperfibrinogenemia, along with hyperviscosity and polyglobuminemia, can augment the hypercoagulable state [7]. These mechanisms can cause cerebrovascular accidents in patients with POEMS syndrome. More cases have been reported regarding limb and coronary artery involvement, but cerebrovascular involvement has been rarely reported [6]. Cerebral infarction has been noted in only two cases who were diagnosed with POEMS syndrome and were on lenalidomidedexamethasone combination therapy [8].

Treatment for POEMS syndrome ranges from radiation, corticosteroids, chemotherapy agents like alkylating agents (melphalan), and immunomodulators (thalidomide and lenalidomide) to targeted therapy with protease inhibitors (bortezomib) and autologous stem cell transplantation. The use of thalidomide, lenalidomide, and bortezomib have shown promising results with significant clinical improvements [9].

Immunomodulators (e.g., thalidomide, lenalidomide) have been associated with thromboembolism. Immunomodulators can increase VEGF, increase the release of cytokines and cause activated protein $\mathrm{C}$ resistance, downregulate thromboplastin, and regulate cyclooxygenase-2 (COX-2) prothrombotic factor. They have been shown to increase the levels of von Willebrand factor and factor VIII, which cause venous and arterial thrombosis [10]. When they are co-administered with corticosteroids, it further increases the risk of thromboembolism [9]. In two phase-3 randomized control trials involving multiple myeloma patients, a combination of lenalidomide with dexamethasone was associated with $3.4 \%$ of cerebrovascular events compared to $1.7 \%$ with dexamethasone alone [11].

In her 2019 update on the various aspects of POEMS syndrome, Angela Dispenzieri did mention prophylactic use of aspirin, as well as the use of low-molecular-weight heparin and warfarin in patients with POEMS syndrome, weighing against their risk factors; however, there have been no studies documenting the prophylactic use of these drugs in this disease so far [3].

\section{Conclusions}

The ischemic event in our patient may have been caused by the combination of POEMS syndrome-associated vasculitis and the thrombogenic nature of lenalidomide-dexamethasone therapy. Further studies are needed to confirm the correlation between vasculitis-induced stroke and lenalidomide-dexamethasone therapy in POEMS syndrome patients. 


\section{Additional Information}

Disclosures

Human subjects: Consent was obtained by all participants in this study. Conflicts of interest: In compliance with the ICMJE uniform disclosure form, all authors declare the following: Payment/services info: All authors have declared that no financial support was received from any organization for the submitted work. Financial relationships: All authors have declared that they have no financial relationships at present or within the previous three years with any organizations that might have an interest in the submitted work. Other relationships: All authors have declared that there are no other relationships or activities that could appear to have influenced the submitted work.

\section{References}

1. Cao CJ, Dou CY, Zhou KH, Liu JB, Lai H: POEMS syndrome presentation with progressive weakness in upper and lower limbs: a case report. Oncol Lett. 2017, 14:5197-5202. 10.3892/ol.2017.6904

2. Nozza A: POEMS syndrome: an update. Mediterr J Hematol Infect Dis. 2017, 9:e2017051. 10.4084/MJHID.2017.051

3. Dispenzieri A: POEMS syndrome: 2019 update on diagnosis, risk-stratification, and management . Am J Hematol. 2019, 94:812-827. 10.1002/ajh.25495

4. Dupont SA, Dispenzieri A, Mauermann ML, Rabinstein AA, Brown RD Jr: Cerebral infarction in POEMS syndrome: incidence, risk factors, and imaging characteristics. Neurology. 2009, 73:1308-1312. 10.1212/WNL.0b013e3181bd136b

5. Sakuta K, Mukai T, Suzuki K, Nishiwaki K, Yaguchi H: Irreversible vasculopathy proceeds rapidly in POEMS syndrome. Intern Med. 2019, 58:3573-3575. 10.2169/internalmedicine.3279-19

6. Rössler M, Kiessling B, Klotz JM, Langohr HD: Recurrent cerebral ischemias due to cerebral vasculitis within the framework of incomplete POEMS syndrome with Castleman disease. (Article in German). Nervenarzt. 2004, 75:790-794. 10.1007/s00115-004-1683-x

7. Yamaguchi I, Satomi J, Yamamoto N, et al.: Coexistence of quasi-moyamoya disease and POEMS syndrome in a patient with intracranial hemorrhage: a case report and literature review. NMC Case Rep J. 2017, 4:5-9. 10.2176/nmccrj.cr.2016-0106

8. Mitsutake A, Matsumoto H, Hatano K, Irie K, Tsukada N, Hashida H: Lenalidomide-induced ischemic cerebrovascular disease in polyneuropathy, organomegaly, endocrinopathy, monoclonal gammopathy, and skin changes syndrome. J Stroke Cerebrovasc Dis. 2018, 27:102-103. 10.1016/j.jstrokecerebrovasdis.2017.12.039

9. Menon SP, Rajkumar SV, Lacy M, Falco P, Palumbo A: Thromboembolic events with lenalidomide-based therapy for multiple myeloma. Cancer. 2008, 112:1522-1528. 10.1002/cncr.23336

10. Palumbo A, Palladino C: Venous and arterial thrombotic risks with thalidomide: evidence and practical guidance. Ther Adv Drug Saf. 2012, 3:255-266. 10.1177/2042098612452291

11. Li W, Cornell RF, Lenihan D, Slosky D, Jagasia M, Piazza G, Moslehi J: Cardiovascular complications of novel multiple myeloma treatments. Circulation. 2016, 133:908-912. 10.1161/CIRCULATIONAHA.115.018351 